\title{
INFLUENCE OF LOCAL ENVIRONMENT ON THE ELECTRONIC STRUCTURE AND MAGNETIC PROPERTIES OF $\mathrm{Ni}_{1-x} \mathrm{Cu}_{x} \mathrm{MnSb}$ ALLOYS
}

\author{
A. JEZIERSKI ${ }^{a}$ AND A. SZYTULA ${ }^{b}$ \\ ${ }^{a}$ Institute of Molecular Physics, Polish Academy of Sciences \\ Smoluchowskiego 17, 60-179 Poznan, Poland \\ b Institute of Physics, Jagiellonian University, Reymonta 4, 30-059 Kraków, Poland
}

The magnetic ordering in $\mathrm{Ni}_{1-x} \mathrm{Cu}_{x} \mathrm{MnSb}$ alloys changes from ferromagnetic $(x=0)$ to antiferromagnetic $(x=1)$. The X-ray and neutron diffraction showed that the systems crystallise in $C 1_{b}$ type structure. We present an influence of the local chemical ordering on the electronic and magnetic properties of $\mathrm{Ni}_{1-x} \mathrm{Cu}_{x} \mathrm{MnSb}$ alloys. The band structure and the magnetic moments were calculated by the spin-polarised linear muffin-tin orbital in the atomic sphere approximation method for the experimental values of the lattice parameters. The magnetic moment of $\mathrm{Mn}$ increases from $3.8 \mu_{\mathrm{B}}$ for $x=0$ to $4.18 \mu_{\mathrm{B}}$ for $x=1$.

PACS numbers: 31.15.Ar, 31.15.Ew, 71.15.Mb, 71.15.-m, 71.15.La, 71.20.-b, 71.20. Be

\section{Introduction}

The electronic and magnetic properties of $\mathrm{Ni}_{1-x} \mathrm{Cu}_{x} \mathrm{MnSb}$ semi Heusler alloys were investigated experimentally and theoretically [1-7]. Particularly, the magneto-optical Kerr effect (MOKE) in half-metallic systems was studied extensively over the last years $[6,7] . \mathrm{Ni}_{1-x} \mathrm{Cu}_{x} \mathrm{MnSb}$ semi Heusler alloys crystallise in $C 1_{b}$ type structure which consists of four interpenetrating fcc sublattices. $\mathrm{Ni}$ and $\mathrm{Cu}$ atoms are distributed randomly in one fcc sublattice with an origin located at $(0.25,0.25,0.25)$. The second fcc sublattice at $(0.75,0.75,0.75)$ is empty. $\mathrm{Mn}$ and $\mathrm{Sb}$ atoms occupy the sublattices located at $(0,0,0)$ and $(0.5,0.5,0.5)$, respectively. Magnetic measurements [1-3] indicated that $\mathrm{NiMnSb}$ was ferromagnetic with $T_{\mathrm{C}}=750 \mathrm{~K}$. Neutron diffraction experiment [3] indicated that CuMnSb was antiferromagnetic with $T_{\mathrm{N}}=55 \mathrm{~K}$. The magnetic moments were alignèd in the ferromagnetic (111) planes with neighbouring planes oriented antiparallel. Szytuła [1] had shown that for $0.6<x<0.95$ the $\mathrm{Ni}_{1-x} \mathrm{Cu}_{x} \mathrm{MnSb}$ had the complex non-collinear magnetic structure. The neutron scattering measurements have shown [1] that the antiferromagnetic contribution to the total magnetic moment decreased to zero for $x=0.6$. Magnetic moment of $\mathrm{Mn}$ in NiMnSb estimated 
from neutron measurements was $3.85 \mu_{\mathrm{B}}$ at $4.2 \mathrm{~K}$ [1]. The lattice parameter $a$ increased almost linearly with the concentration from $a=0.5909 \mathrm{~nm}$ for $x=0$ to $a=0.6095 \mathrm{~nm}$ for $x=1$ [1]. In this work we present an $a b$ initio study of the influence of local chemical ordering in $\mathrm{Ni}-\mathrm{Cu}$ sublattice on the electronic and magnetic properties of $\mathrm{Ni}_{1-x} \mathrm{Cu}_{x} \mathrm{MnSb}$ alloys.

\section{Method of calculation and results}

The electronic structure and magnetic moments were calculated by the self-consistent tight binding linear muffin-tin orbital (TB LMTO) method [8] within the framework of the local spin density (LSD) approximation. The scalar-relativistic approximation for the band electrons and the fully relativistic treatment for the frozen core electrons were applied. The exchange correlation potential was taken in the form proposed by von Barth and Hedin [9] and the gradient corrections were applied [10]. The values of the atomic spheres radii in the atomic sphere approximation (ASA) were taken in such a way that the sum of the all atomic sphere volumes was equal to the volume of the unit cell. The values of the atomic potential for each atoms were the same at the sphere boundary. The band structures were carried out for more than $260 k$-points in the irreducible wedge of the Brillouin zone. The number of $k$-point depended on the symmetry of the system. In order to study the effect of the local environment we replaced each fcc sublattice by four simple cubic sublattices. In such a way we observed the change of the electronic structure and the values of the magnetic moments when the distribution of nickel and copper atoms in sublattices was changed. The self-consistent band calculations were performed for the paramagnetic, ferromagnetic phase and also for the case in

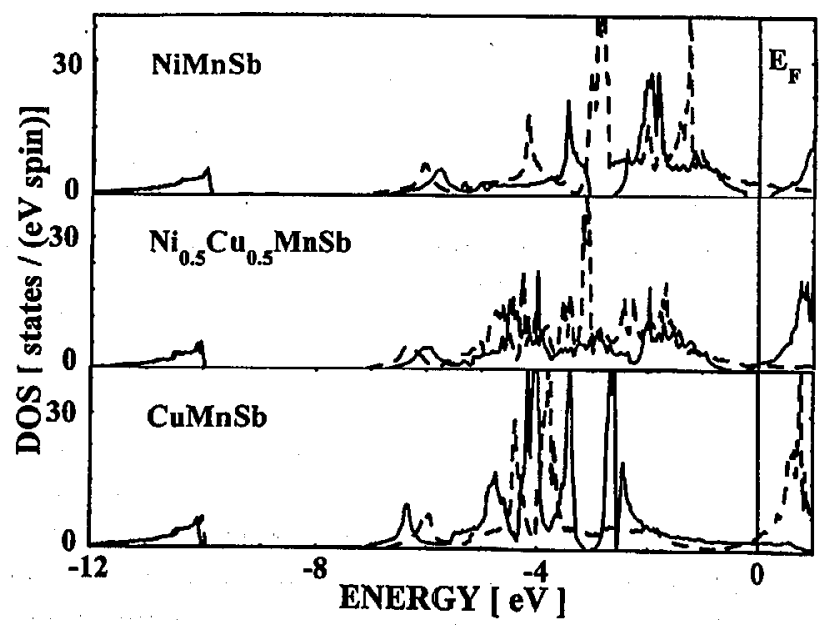

Fig. 1. Electronic density of states for the ferromagnetic $\mathrm{Ni}_{1-x} \mathrm{Cu}_{x} \mathrm{MnSb}$. The Fermi level is located at $E=0 \mathrm{eV}$. The solid (broken) curve presents the DOS for spin down (up) direction. 
which two sc sublattices occupied by manganese atoms had the opposite direction of the magnetic moments.

In Fig. 1 we presented the electronic density of states (DOS) for the ferromagnetic $\mathrm{Ni}_{1-x} \mathrm{Cu}_{x} \mathrm{MnSb}$ alloys. The solid (broken) curres show the DOS for spin-down and spin-up, respectively. The Fermi level is located at $0 \mathrm{eV}$. For NiMnSn in spin-down density of states there is a gap at the Fermi level. The substitution of nickel by copper atom decreases the gap. The shape of DOS for $\mathrm{NiMnSb}$ is similar to those obtained by Kulatov and Mazin [4].

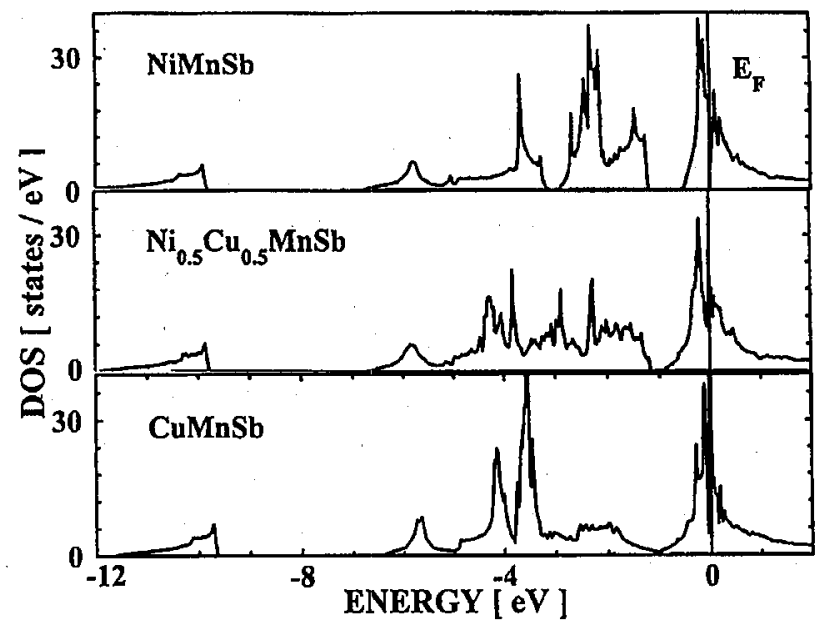

Fig. 2. Electronic density of states for the paramagnetic $\mathrm{Ni}_{1-x} \mathrm{Cu}_{x} \mathrm{MnSb}$. The Fermi level is located at $E=0 \mathrm{eV}$.

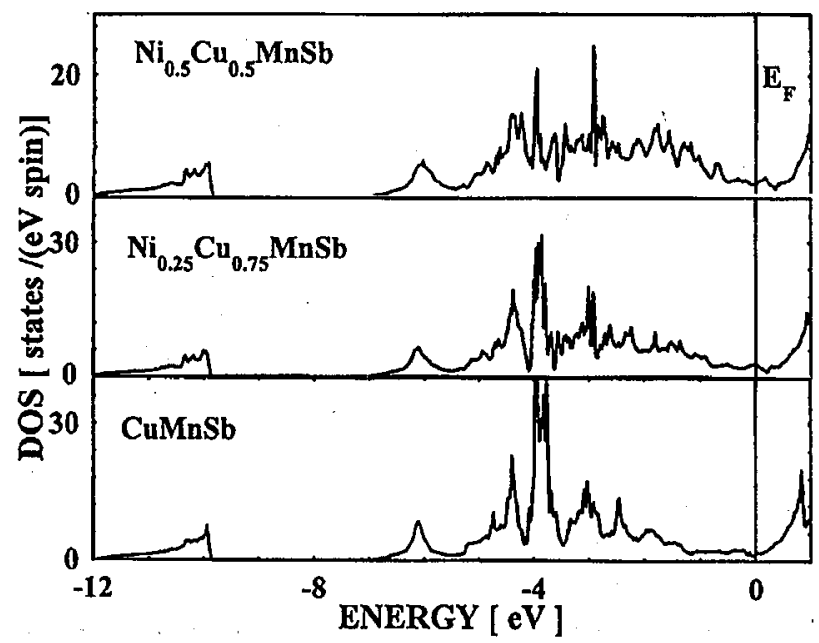

Fig. 3. Electronic density of states for the antiferromagnetic $\mathrm{Ni}_{1-x} \mathrm{Cu}_{x} \mathrm{MnSb}$. The Fermi level is located at $E=0 \mathrm{eV}$. 
In the paramagnetic phase the Fermi level is located in the manganese $d$-band (Fig. 2). The gap below $E_{\mathrm{F}}$ decreases with the increase in copper concentration. The copper and nickel atoms can be distributed randomly in four sc sublattices. We performed the spin-polarized self-consistent band calculations for the different distribution of copper and nickel atoms in the sublattices. Our ab initio calculations indicated that the local disorder in the $\mathrm{Ni}-\mathrm{Cu}$ sublattices led to increase in the total energy and the system became unstable. The manganese atoms occupy in $C 1_{b}$ structure four simple cubic sublattices. In the ferromagnetic phase all magnetic moments of manganese are parallel. We recalculated the band structure of $\mathrm{Ni}_{1-x} \mathrm{Cu}_{x} \mathrm{MnSb}$ for $0.5<x<1$ for antiparallel oriented manganese moments in two sc sublattices.

The total density of states for the antiferromagnetic ordering is presented in Fig. 3. The main peak from $3 d$ manganese is located near $E=-4 \mathrm{eV}$. The density of states at the Fermi level is small for the antiferromagnetic CuMnSb. Our self-consistent band calculations have shown that the antiferromagnetic ordering is more stable than the ferromagnetic ones.

\section{Conclusions}

In this paper we presented ab initio TB LMTO calculations of the electronic and magnetic properties of $\mathrm{Ni}_{1-x} \mathrm{Cu}_{x} \mathrm{MnSb}$ alloys. Our results have shown that the substitution of nickel by copper atom influences the value of the magnetic moment of manganese. The local disorder in $\mathrm{Ni}-\mathrm{Cu}$ sublattice led to decrease in the magnetic moment of manganese.

\section{Acknowledgments}

One of us (A.J.) thanks the Committee for Scientific Research for financial support (project No. 2PO3B 027 15).

\section{References}

[1] A. Szytuła, Acta Phys. Pol. A 43, 787 (1973).

[2] K. Endo, J. Phys. Soc. Japan 29, 643 (1970).

[3] R.H. Forster, G.B. Johnston, J. Phys. Chem. Solids 29, 855 (1968).

[4] E. Kulatov, I.I. Mazin, J. Phys., Condens. Matter 2, 343 (1990).

[5] S.J. Youn, B.I. Min, Phys. Rev. B 51, 10436 (1995).

[6] V.N. Antonov, P.M. Oppeneer, A.N. Yaresko, A.Ya. Perlov, T. Kraft, Phys. Rev. $B$ 56, 13012 (1997).

[7] R.A. de Groot, K.H.J. Buschow, J. Magn. Magn. Mater. 54-57, 1377 (1986).

[8] O.K. Andersen, O. Jepsen, Phys. Rev. Lett. 53, 2571 (1984).

[9] U. von Barth, L. Hedin, J. Phys. C 5, 1629 (1972).

[10] D. Hu, D.C. Langreth, Phys. Scr. 32, 391 (1985). 